\title{
Child Marriage and Female Educational Attainment: A Complex Relationship
}

\author{
Maria Polyakova ${ }^{1}$ (D)
}

Published online: 23 December 2018

(C) The Author(s) 2018

JEL 010

Recently there had been increased interest worldwide in the issue of child marriage due to connections with gender equality and economic development problems. It is a violation of fundamental human rights which also stalls development and perpetuates poverty and inequality at the country and regional levels. Despite this, child marriage rates remain high and investment in ending this practice remains limited.

Research is needed that proves the detrimental effects child marriage may have. This paper focused on one of the main channels: the educational deprivation of child brides. Prior research in the area was limited, but found a significant negative relationship between child marriage and educational attainment. However, an endogeneity problem existed. The decision was often either to get married or continue with education. Thus, unobservable factors such as individual ability, cultural beliefs and economic concerns, may have been present in the error term. Prior literature has either failed to account for this problem or used instruments of questionable validity.

The above creates ample motivation for another investigation into the relationship between child marriage and educational attainment. Using a new data set and a slightly different estimation strategy, this paper both investigated whether the instruments used previously were valid and attempted to establish a causal relationship between child marriage and educational attainment.

The study data source was the 2013 Demographic and Health Survey for Nigeria. It was primarily selected due to data availability and the high rate of child marriage. Thirty-nine percent of girls marry before the age of 18 (Women Living Under Muslim Laws, A Submission to the UN Office of the High Commissioner on Human Rights, 2013). There was also significant geographical disparity. In the northern states of

Maria Polyakova

polyakova.maria.a.97@gmail.com

1 Department of Economics, The University of Warwick, Coventry, UK 
Nigeria, the percentage of girls who married before age 18 was approximately twice the national average (Brown, UNESCO Report, 2012).

Three different regression specifications were constructed in order to carry out the analysis. The first was a simple probit equation, followed by two IV-probit specifications with two different sets of instrumental variables. These equations were run for two different dependent binary variables: one for whether the female had at least some secondary education and one for whether the female had completed secondary education. The main independent variable of interest was the number of years of child marriage. A number of individual-level control variables were introduced and Primary Sampling Unit (PSU)-level control variables were also introduced.

The two sets of instrumental variables used were modelled on those employed in previous literature. The first included PSU-level variables measuring contemporaneous and past (age group 34-49) leave-out-mean incidence of child marriage for each age of marriage (meaning share of those married at a certain age was calculated for all individuals in the PSU except for the one considered in the regression). The second set of instrumental variables used past (age group 34-49) leave-out-mean incidence of child marriage for each age of marriage, proportion of those who have only engaged in sex after marriage and the average total number of children in the PSU. These variables were seen as capturing factors such as social norms that may affect child marriage, but not the decision to continue with education.

The results for all equations were significant even at the $1 \%$ level. However, the null hypothesis was rejected for the child marriage variable being exogenous. Thus, the IVprobit specifications may be seen as providing more reliable results. Compared to the non-IV equation, the results were higher. The two IV-specifications suggested a similar magnitude of the effect: delaying marriage by a year was associated with an $8.9 \%$ point increase in the probability of having some secondary education. It was also associated with a $10-11 \%$ point increase in the probability of completing secondary school. However, in overidentification testing, the zero hypothesis of no overidentification was rejected for both IV-specifications. Thus, in both cases, the validity of the instruments, and therefore the reliability of the results, was questionable.

No conclusions as to the magnitude of the relationship could be drawn, since the endogeneity problem could not be solved. However, the work still confirmed past research results, both regarding the endogeneity problem and the effect of child marriage on educational attainment. Perhaps the biggest contribution of this paper is that it casts doubt on the relevance of two of the three IV-estimation strategies used in previous research. This provides motivation for future research.

The current results suggest the existence of a significant correlation between the decision to marry early and the decision to leave school. Thus, investment in policies aimed at solving this issue is indeed necessary.

Open Access This article is distributed under the terms of the Creative Commons Attribution 4.0 International License (http://creativecommons.org/licenses/by/4.0/), which permits unrestricted use, distribution, and reproduction in any medium, provided you give appropriate credit to the original author(s) and the source, provide a link to the Creative Commons license, and indicate if changes were made.

Publisher's Note Springer Nature remains neutral with regard to jurisdictional claims in published maps and institutional affiliations. 